\title{
Gallium (III) Salophen as a Dual Inhibitor of Pseudomonas aeruginosa Heme Sensing and Iron Acquisition
}

Garrick Centola, Daniel J. Deredge, Kellie Hom, Yong Ai, Alecia T. Dent, Fengtian Xue*, Angela Wilks*

Department of Pharmaceutical Sciences, University of Maryland Baltimore, Baltimore MD 21201, United States

*Corresponding Author Email: fxue@rx.umaryland.edu; awilks@rx.umaryland.edu

\section{Table of Contents:}

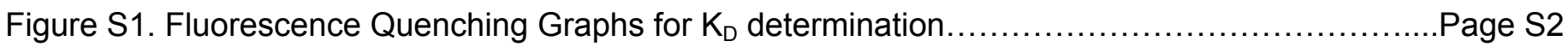

Figure S2. Heme Receptor Mutant Controls Supplemented with Holo-HasAp and $\mathrm{FeCl}_{3} \ldots \ldots \ldots \ldots . .$. Page S2

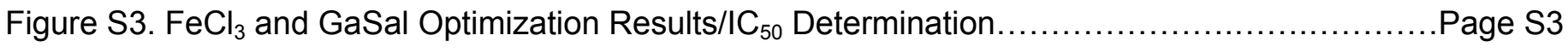

Figure S4. Pyoverdine Biosynthesis Deletion Strain Growth Curves with FeSal, GaSal...............Page S3

Figure S5. Coverage Map for Wild-Type Full-Length HasAp used for HDX-MS ......................Page S4

Figure S6. Surface Plasmon Resonance Traces and Steady-State Binding Fit......................Page S4 

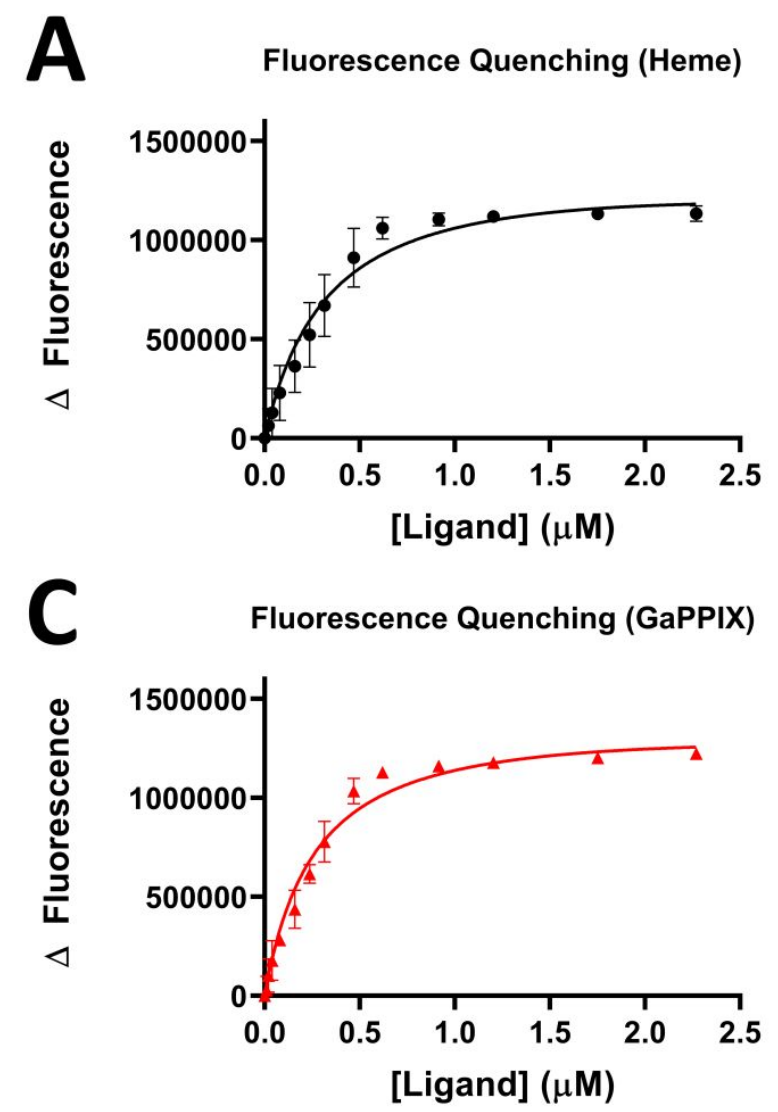

B

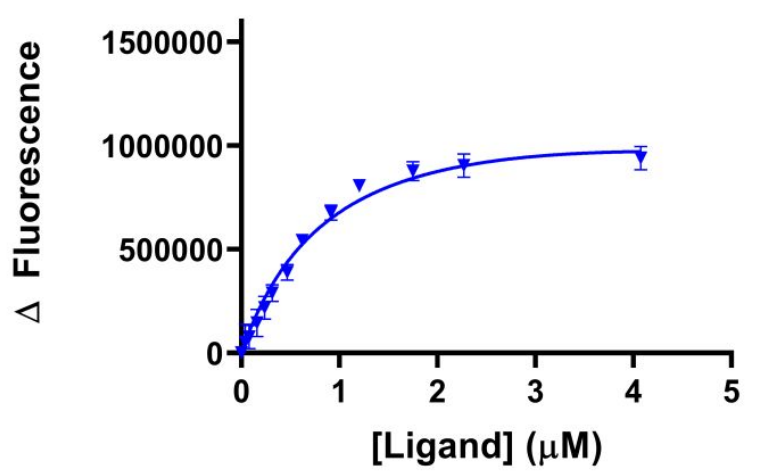

Fluorescence Quenching (GaSal)

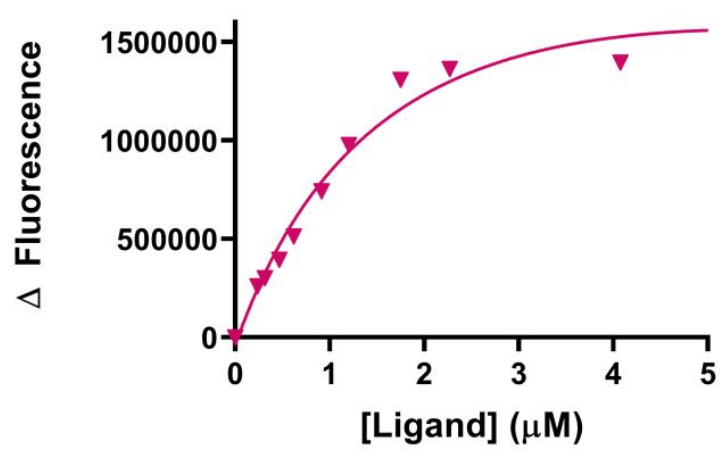

Figure S1. Fluorescence Quenching Graphs for $K_{D}$ Determination. To $1 \mu M$ apo-HasAp in 20 mM sodium phosphate buffer $\left(\mathrm{pH} 7.4,25^{\circ} \mathrm{C}\right)$ was added the ligand of interest until saturation was observed. The binding constant $\left(\mathrm{K}_{\mathrm{D}}\right)$ determined by fitting data to a one-site binding model in Graphpad Prism8 according to the decrease in fluorescence maximum as a function of increasing ligand concentration, accounting for dilution upon titration. Data represent the average of three replicates.

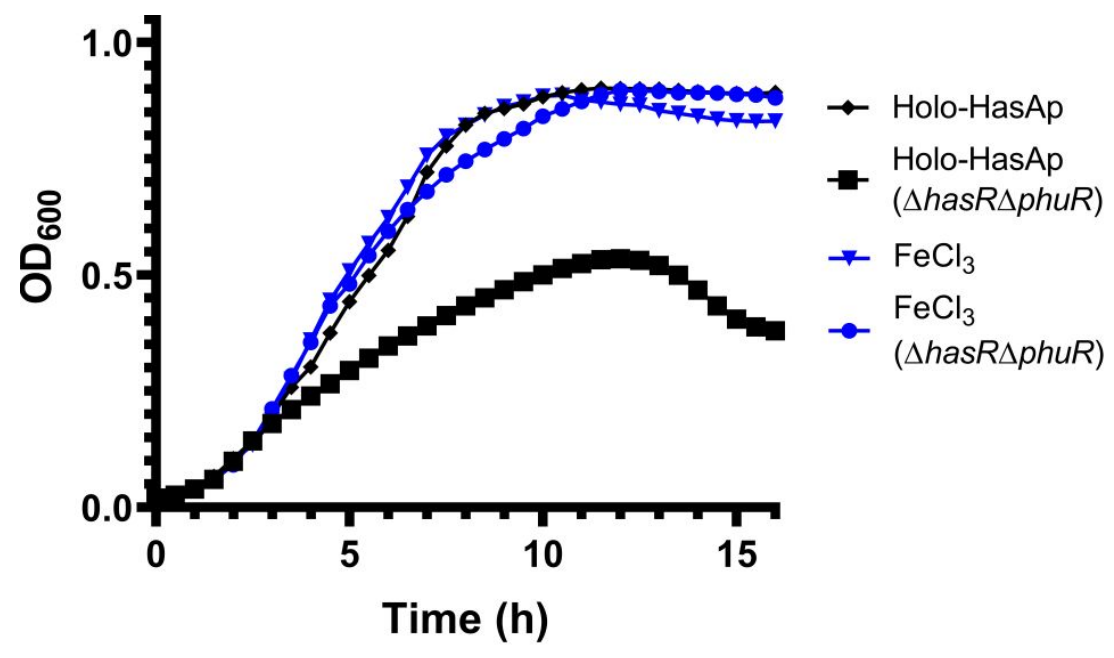

Figure S2. PAO1 $\Delta$ hasR $\Delta$ phuR Heme Receptor Deletion Controls. Controls indicate growth is not impacted by the deletion of the heme receptors in the presence of $\mathrm{FeCl}_{3}$ whereas heme uptake from HoloHasAp is reduced in the mutant strain. Cultures were prepared in M9 minimal media and $1 \mu \mathrm{M}$ supplements added as outlined in the Methods section. Data represent the average of three independent cultures following subtraction of blank (cell-free) samples to account for background absorbance. 
PA01 $\Delta$ hasAp

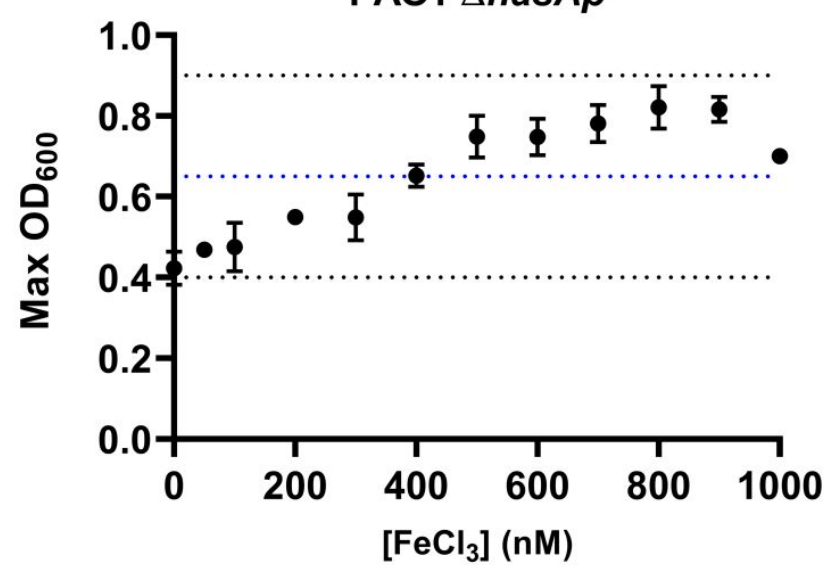

PAO1 $\Delta$ hasAp + $400 \mathrm{nM} \mathrm{FeCl} 3$

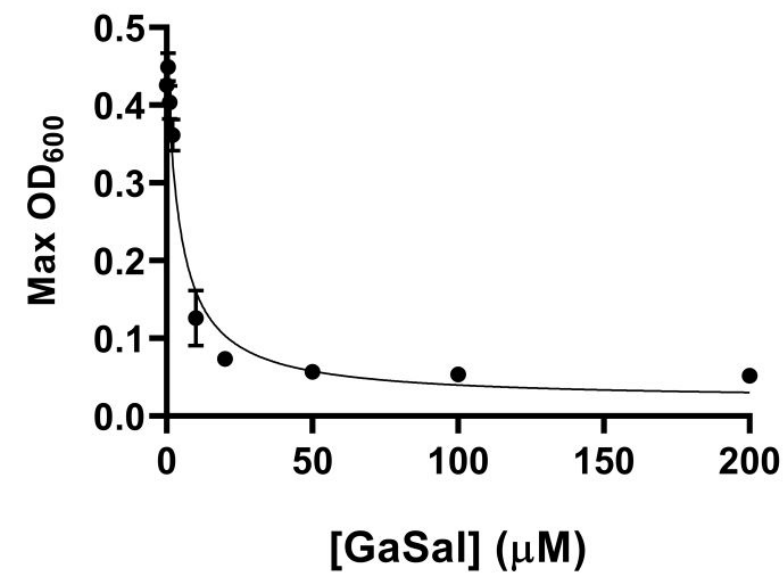

Figure S3. PA01 $\Delta$ hasAp Titration Optimization Results. A) Titration with $\mathrm{FeCl}_{3}$. B) Titration with GaSal. Cultures were prepared in M9 minimal media and $1 \mu \mathrm{M}$ supplements added as outlined in the Methods section. Data represent the average of three independent cultures following subtraction of blank (cell-free) samples. Maximum OD600 values were recorded after $16 \mathrm{~h}$ growth

A

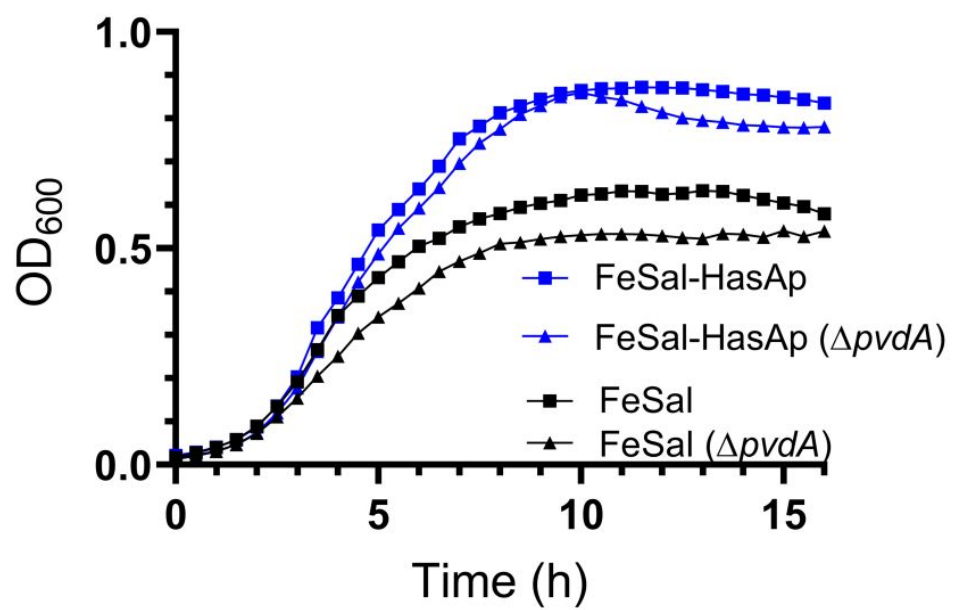

FeSal Comparison

B

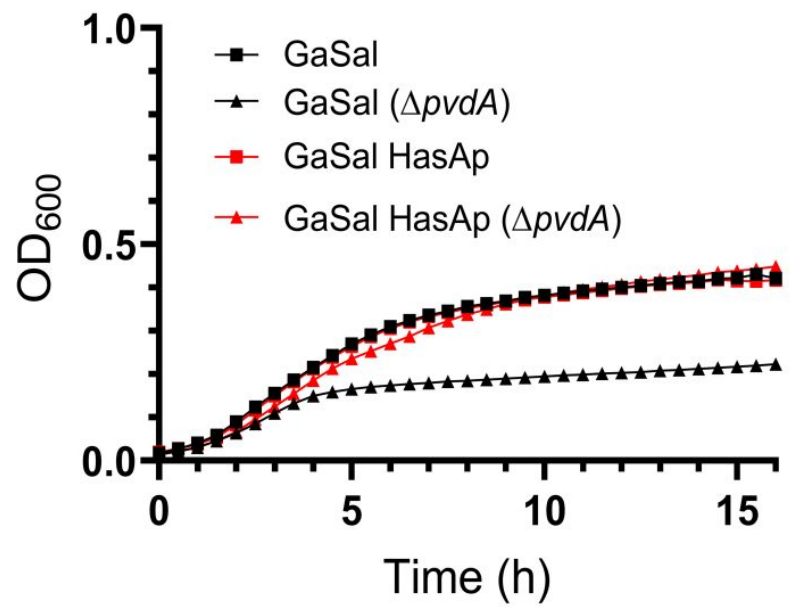

Figure S4. PA01 $\triangle p v d A$ Pyoverdine Biosynthesis Deletion Strain Growth Curves. The lack of the higheraffinity siderohore, pyoverdine, does not reduce the use of FeSal as an iron source $(A)$ or prevent gallium toxicity with GaSal (B), further showing that the metal center is not stripped by siderophores and the complex is taken up as intact salophen. Cultures were prepared in M9 minimal media and $1 \mu \mathrm{M}$ supplements added as outlined in the Methods section. Data represent the average of three independent cultures following subtraction of blank (cell-free) samples to account for background absorbance. 


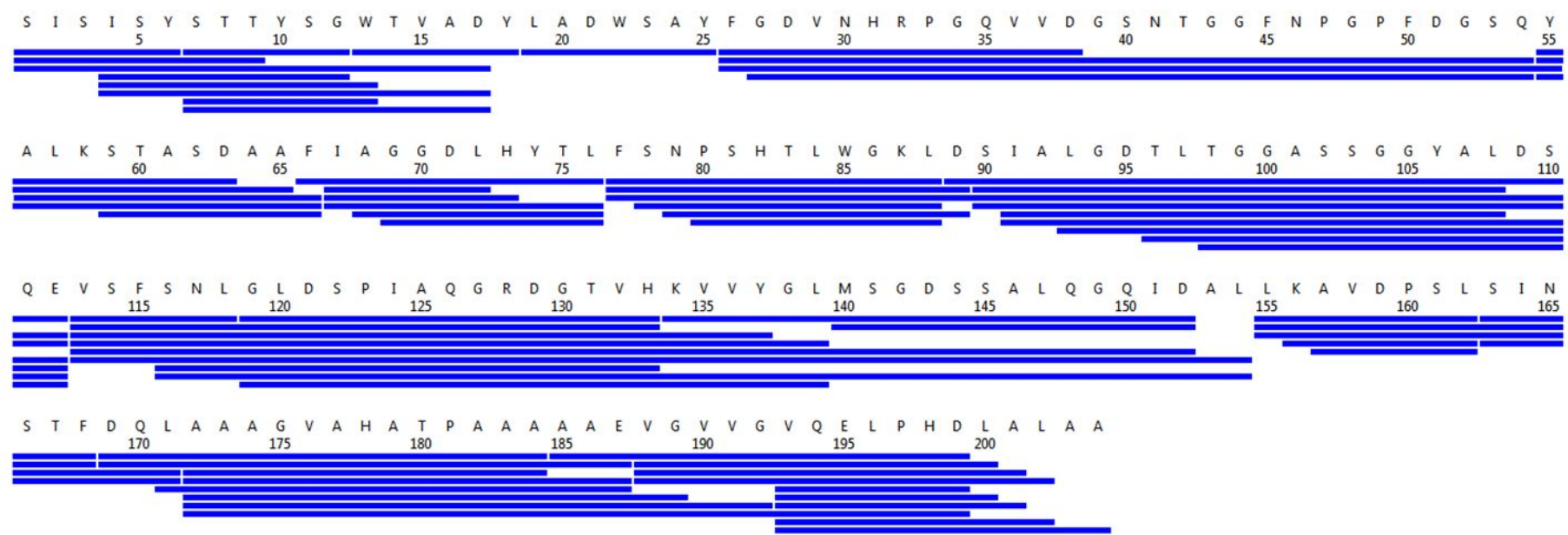

Total: 76 Peptides, $100.0 \%$ Coverage, 5.68 Redundancy

Figure S5. Coverage Map of Wild-Type Full-Length HasAp Following Pepsin Digestion. The coverage map was obtained as described in the experimental methods section. Complete coverage of the protein sequence was achieved over 76 peptides with an average redundancy of 5.7 peptides/residue.

A.
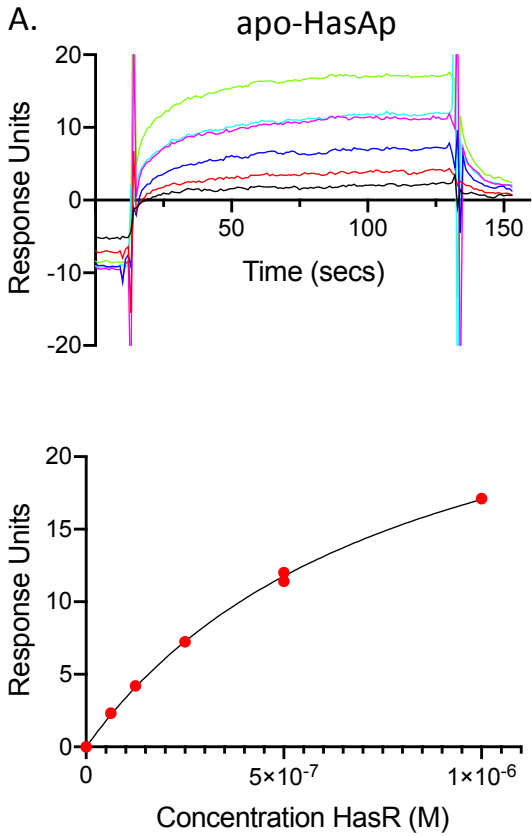

B.
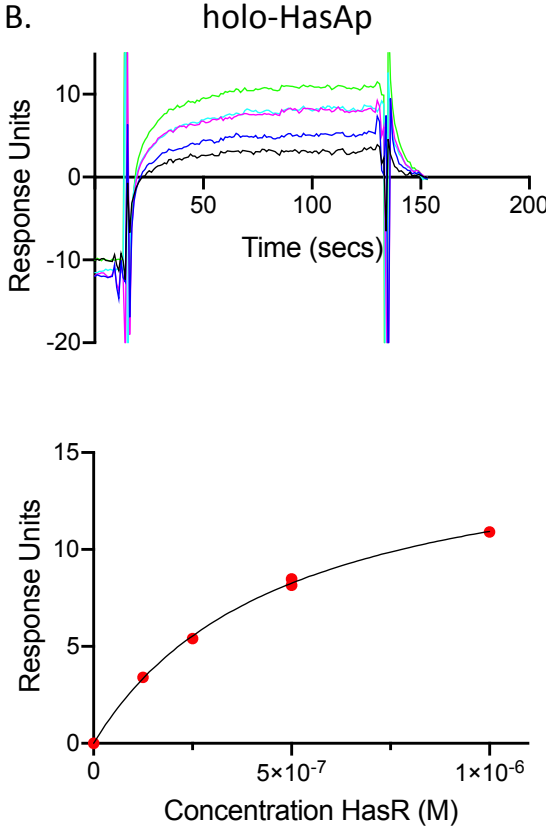

C. GaSal-HasAp
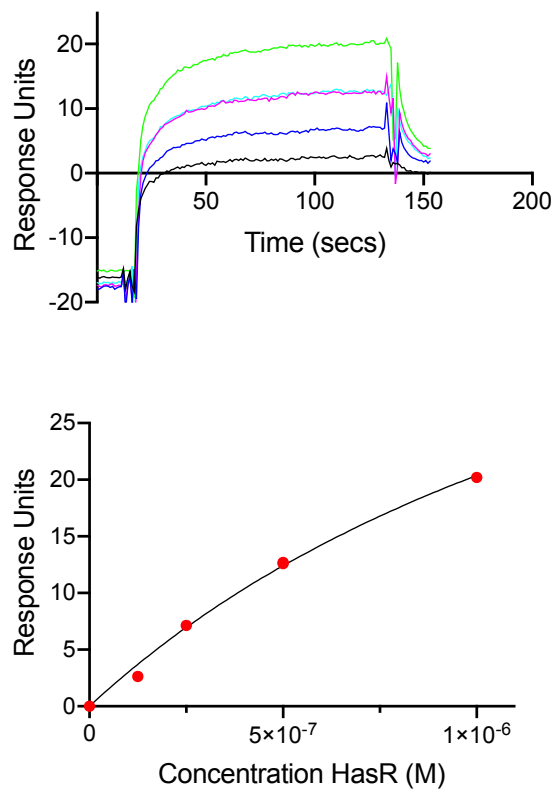

Figure S6. Surface Plasmon Resonance Traces and Steady-State Binding Fit. HasAp was directly immobilized to the chip and HasR was used as the analyte at concentrations from 0.063 to $1 \mu \mathrm{M}$.

Representative sensorgrams are shown in the upper panel. Response differentials at steady state were plotted as a function of analyte concentration and fit to a 1:1 binding model as shown in the lower panel. To obtain a more appropriate fit, $\mathrm{K}_{\mathrm{D}}$ values were obtained from steady-state response values as a function of analyte concentration $\left(\mathrm{X}^{2}=0.02-0.5\right)$ for $n=3$. A) apo-HasAp B) holo-HasAp C) GaSal-HasAp. 